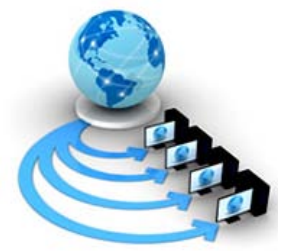

International Journal of Advanced Research in Computer Science

RESEARCH PAPER

\title{
SMART AGRICULTURE SYSTEM + AND IMAGE PROCESSING
}

\author{
Snowber Mushtaq \\ Department of Computer science \\ National Institute of technology Srinagar \\ Srinagar, Jammu and Kashmir, India
}

\begin{abstract}
As we know Indians most of the population is dependent on agriculture for their livelihood. So we need to integrate the IoT technology and image processing technology with our agriculture so as to increase the growth of agriculture, which in turn strengthens the economy of our country. The main aim of the paper is to combine image processing and IoT based technology for developing smart agriculture based system. The virtue is to gather real time data of agriculture domain using cameras and sensors and send that data through message to farmers. Here we present a smart agriculture system based on IoT and supported with internet technology and sends collected information to farmers. Internet of Things (IoT) is believed to be one of the fastest developing technologies and of research interest in computer science.
\end{abstract}

Keywords: Smart agriculture, IOT, Sensor technology, Image processing, Cloud computing, Wi-Fi.

\section{INTRODUCTION}

About $70 \%$ of Indian population is dependent on agriculture. The rate of crop production has declined since 2010 due to various weather conditions, crop disease, rodents, fertilizer misuse or low soil fertility etc. It is very essential to take steps for growth of agriculture as Indian economy and livelihood is dependent on agriculture. It is very essential to change a way of advancement of agriculture, IoT based technology and its integration with image processing technology provides solution to it. It includes cameras which capture images at regular time period and image recognition techniques to recognize disease, rodents and growth of crops. The CCTV cameras are installed at various places in crop fields and grain reservoir. The exchange of information due to IoT (Internet of Things) can be achieved at reasonable cost. The sensors are also implemented at various places in the crop field and grain field they collect information about various things like moisture in soil, temperature, humidity and various agriculture parameters. The information transmission and analysis from various sensors and cameras are done by integration of internet of things. The crop loss due to crop disease, soil moisture or waste water before pre-harvest and loss due to rodents in grain store can be diminished.

The image processing techniques include various mathematical and computer algorithms performed on images for image classification, extraction of patterns and feature extraction. It has allowed a vast domain of algorithms to be applied on input image and it includes techniques that can remove problems like image distortion and noise. It includes various fourier transformation techniques and filtering techniques on original images for identification of rodents and identification of disease in a crop.

Internet of things include communication technology ,information to be transmitted, updated infrastructure and networks which is able to connect all the information collecting devices and is able to connect them for information transmission. In the agriculture field we know, we have an extensive area to analyze. So we need to have sensors to collect information and observe domain of agriculture field and pass analyzed information to farmers through internet.

\section{Litrature REVIEW}

The system that we need to develop needs first to collect data ,analyze data and carry information over network to remote location and intensify it by combining it with new technology .Today we have IP based cameras and we need to connect them to internet .So as to monitor continuously our remote agriculture field. We need to integrate them with computational ability like detecting crop disease, rodents ,monitor growth of crop in the field and notify information by activating electronic devices and raspberry pi and transmitting information to server, which in turn sends information to farmers.

For implementing the system we can have either two or five layer architecture [1]. In three layer architecture, first layer is perception layer which is meant for collection of information from various sensors and cameras. Second layer is the network layer which is meant for information transmission over the network and it has computational capability. Third layer is the application layer which has practical applications like sending sms to the end users.

This system can be developed using python computer programming language and the scripts written in this language are used to control and monitor the information from cameras and sensors. The advantages of the system are: it provides security from rodents. It can identify the rodents, mammals, humans. Cameras can be moved in various directions based on the commands.

We also need to address factors that impact the health of a plant like humidity, moisture content of soil, intensity of light. The growth of a plant can be monitored by the cameras, morphological change of plant are continuously monitored [2]. The components needed here include soil moisture sensor, DHT11 which is moisture and humidity sensor, camera module for capturing images at regular intervals. First the sensors are activated then after images are captured. So as to study how humidity, moisture content in soil, and intensity of 
light effect the plant growth. And it is the cost effect way of studying how various factors affect the plant growth. We can keep images and data in SD card for further analysis. Here the aim is to find the factors that affect the plant growth. It can help farmers as they can't monitor the field throughout the day. And the information collected can be used for further study.

The crop disease can be identified by various image processing techniques and alerts can be send to farmers [3] and it is possible control the field remotely. We can develop any time any where web and mobile application. The system can be divided into three modules namely client module, server module and farm module. The farm module includes sensing information about agriculture parameters, location identification of sensors, transferring information to processing module for decision making and monitoring of agriculture field using cameras. The system can be supported with solar panels for energy source to sensors and server. Ubisense mote is sensor board including humidity, light intensity, barometric pressure and buzzers. The cameras an also be integrated, it produces alarms through buzzers. The system obtains weather related information from internet, so as to keep the appropriate amount of water in the field.

Need of agriculture is going to increase because of increase in population; agriculture is the basic source of food to all humans. And it gives employment opportunity to lot of people in the world [4].We can use gps for message transmission to the farmers. CC3200 is a single chip with embedded microcontroller, Wi-Fi and network processing unit. It is a low cost computational device for connecting sensors and works asynchronously for transmission and reception of information. The power supply can be provided through small portable batteries.

Internet of things is needed to implement in agriculture for modernization of our agriculture field as is known as Agro Tick [5]. It includes 1) IoT: it includes embedded hardware, FPGA based design, ASIC/SOCIP, broad level. 2) Big Data Analytics: NLP, predictive analysis, machine learning. 3) Software: Android app, multilayered firmware, cloud platform, secure API's. In the current modern era our agriculture field is blessed with growth of technology in the field of Information technology, computers and electronics. Agriculture in combination with IoT, Big Data Analytics, cloud computing can lead to tremendous growth of agriculture in future.

Based on the calculation of agricultural parameters, we can have precision agriculture. It has played a vital role in the growth of agriculture in developing countries. Previously, GPS (Global Packet Service) and GIS (Geographical Information System) were used for precision agriculture but they were costly [6]. So there was a need to develop a cost effective system. The introduction of wireless communication technology, sensors and CCTV cameras led to cost affective system. With the evolvement of graphical user interface the growth of the crop can be monitored remotely for a longer period of time. And this can be used for the potential growth of the new crop field. In the traditional system farmers used to monitor the agriculture field for deciding about when to irrigate, when to applying require fertilizers, pesticides. They used to follow a mental model which does not give always perfect results and they sometimes used to fail. We propose a wireless cameras and sensor based crop field monitoring and management system. The system need to be integrated with self powered with solar panels or small batteries and long performance cameras. The advantages of the system include the lower cost of implementation of sensor nodes and IoT technology. The only thing we need to keep into consideration is to protect sensors from moisture, heat or any damage.

With the development of Cyber-physical farm management cycle has played a vital role in socio-economical growth [7]. It includes Internet of things, cloud computing, big data and Artificial intelligence in our farming. The large volume of information can be captured through big data analytics and stored on cloud. The big data has recent development and it has a vast application as everything in IoT is in the form of data and needs to be managed for analysis and decision making.

The digital earth concept is incomplete without digital agriculture. Agriculture information system is also known as intelligent agriculture or information agriculture [8], which has evolved from farm land cultivation, farm land information, agriculture engineering equipments, agriculture chemical control. In the twenty first century there is a need for developing intelligent agriculture for saving water, and producing pollution free, high yield and high quality agricultural products. The modernization concept includes digitalization in every aspect of agriculture. Agriculture modernization has been accepted in developed countries like United States. Intelligent farming helps in selecting which seed will grow better in which field, so as to get the maximum benefit. In our country there is a need for developing such a system as most of the population is dependent on agriculture for their livelihood.

Smart robots can be incorporated in our field and they can perform tasks like animal scaring, irrigating, spraying weedicides, pesticides, moisture sensing etc [9]. Different sensors are connected through raspberry pi and wireless communication can be achieved between different sensors and server.

\section{SYSTEM OVERVIEW}

The proposed system consists of four main modules: 1) Data Collection Module: It consists of various set of sensors for humidity, temperature, pressure, intensity of light, ph value of soil ,water level measurement and set of cameras places at regular intervals for capturing images and videos. 2) Gateway Module: This module acts as a connector for connecting various sensors and cameras by wireless communication. 3) Cloud Module: It is a data storage module here data is collected compared and analyzed for decision making.. After decision making the control information is send to end users (farmers) through web and mobile applications. 


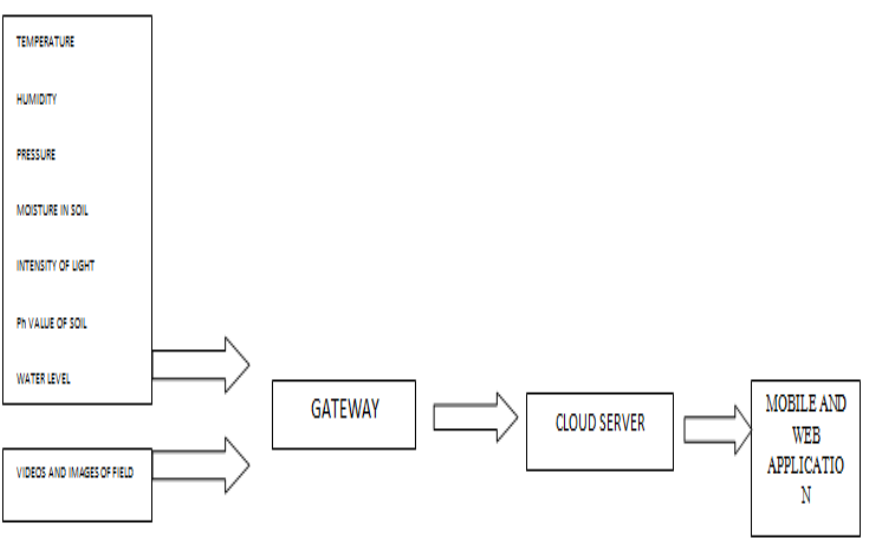

Figure 1:IoT Block Diagram

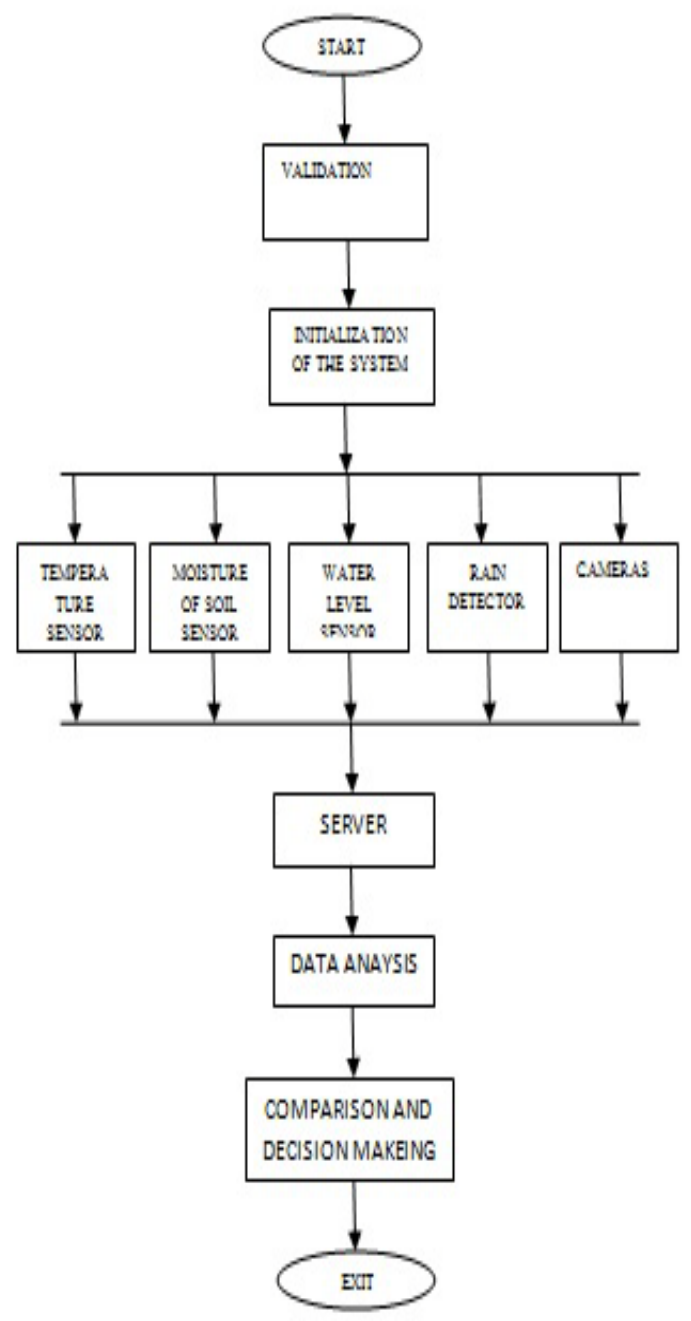

Figure 2:Flow Chart Of Proposed System

The proposed system works on the basis of user validation. When the user validates the system all the connected sensors and cameras are activated and the information is send to cloud server for comparison with the previous results and decision making and the farmers are informed through web and mobile applications.

\section{CONCLUSION}

Growth of crops is the major challenge in our country as most population is dependent on agriculture. The incorporation this smart technology in our agricultural field can lead to the digital green revolution. So for the growth of our agriculture we need to incorporate Internet of Things, cloud computing and sensors in our fields.

\section{REFRENCES}

[1] T. Baranwal, Nitika and P. K. Pateriya, "Development of IoT based smart security and monitoring devices for agriculture," 2016 6th International Conference - Cloud System and Big Data Engineering (Confluence), Noida, 2016, pp. 597-602.

[2] A. Kapoor, S. I. Bhat, S. Shidnal and A. Mehra, "Implementation of IoT (Internet of Things) and Image processing in smart agriculture," 2016 International Conference on Computation System and Information Technology for Sustainable Solutions (CSITSS), Bangalore, 2016, pp. 21-26.

[3] C. Cambra, S. Sendra, J. Lloret and L. Garcia, "An IoT service-oriented system for agriculture monitoring," 2017 IEEE International Conference on Communications (ICC), Paris, 2017, pp. 1-6.

[4] S. R. Prathibha, A. Hongal and M. P. Jyothi, "IOT Based Monitoring System in Smart Agriculture," 2017 International Conference on Recent Advances in Electronics and Communication Technology (ICRAECT), Bangalore, 2017, pp. 81-84.

[5] S. Roy et al., "IoT, big data science \& analytics, cloud computing and mobile app based hybrid system for smart agriculture," 2017 8th Annual Industrial Automation and Electromechanical Engineering Conference (IEMECON), Bangkok, 2017, pp. 303-304.

[6] M. S. Mekala and P. Viswanathan, "A novel technology for smart agriculture based on IoT with cloud computing," 2017 International Conference on I-SMAC (IoT in Social, Mobile, Analytics and Cloud) (I-SMAC), Palladam, 2017, pp. 75-82.

[7] Sjaak Wolfert, Lan Ge, Cor Verdouw, Marc-Jeroen Bogaardt, Big Data in Smart Farming - A review, In Agricultural Systems, Volume 153, 2017, Pages 69-80, ISSN 0308-521X.

[8] D. Yan-e, "Design of Intelligent Agriculture Management Information System Based on IoT," 2011 Fourth International Conference on Intelligent Computation Technology and Automation, Shenzhen, Guangdong, 2011, pp. 1045-1049.

[9] Nikesh Gondchawar1, Prof. Dr. R. S. Kawitkar2, "IoT based Smart Agriculture" International Journal of Advanced Research in Computer and Communication Engineering Vol. 5, Issue 6, June 2016 